\title{
Alteration of lens and retina textures from mice embryos with folic acid deficiency: Image processing analysis.
}

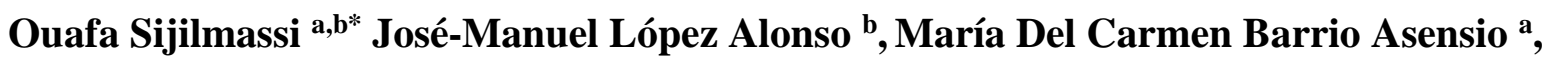 \\ Aurora Del Río Sevilla ${ }^{\text {a }}$ \\ ${ }^{a}$ Universidad Complutense De Madrid, Faculty of Optics and Optometry, Anatomy and Human Embryology \\ Department, Avda. Arcos de Jalón, 118, Madrid, Spain, 28037. \\ ${ }^{\mathrm{b}}$ Universidad Complutense De Madrid, Faculty of Optics and Optometry, Optics Department, Avda. Arcos de \\ Jalón, 118, Madrid, Spain, 28037.
}

*E-mail: o.sijilmassi@ucm.es

Telephone number: +34 913946874

ORCID of the authors:

Ouafa Sijilmassi: 0000-0003-0513-0229

José Manuel López Alonso: 0000-0001-6349-1845

María del Carmen Barrio Asensio: 0000-0002-8475-2642

Aurora del Río Sevilla: 0000-0001-7619-8656

\begin{abstract}
:
Purpose: Folic Acid (FA) is an essential vitamin for embryonic development. It plays particularly a critical role in RNA, DNA, and protein synthesis. On the other hand, the collagen IV and laminin-1 are important proteins during embryonic development. This study was done to find if FA-deficiency at a short and a long-term in mothers could alter the tissue texture of retina and lens of the progeny.

Methods: Collagen IV and laminin-1 were localized by immunohistochemistry in the lens and retina of the FA-deficient embryos. To carry out the image processing, texture segmentation was performed through canny edge detection and Fourier transform (FT). We defined a parameter, the grain size, to describe the texture of the lens and retina. A bootstrap method to estimate the distribution and confidence intervals of the mean, standard deviation, skewness and kurtosis of the grain size has been developed.

Results: Analysis through image processing using Matlab showed changes in the grain size between control and FA deficient groups in both studied molecules. Measures of texture based on FT exhibited changes in the directionality and arrangements of type IV collagen and laminin-1.

Conclusions: Changes introduced by FA deficiency were visible in the short-term (two weeks) and evident in the long term (eight weeks) in both grain size and orientation of fibre structures in the tissues analysed (lens and retina). This is the first work devoted to study the effect of FA deficit in the texture of eye tissues using image processing techniques.
\end{abstract}

Keywords: Folic acid deficiency, type IV collagen, laminin-1, Matlab texture analysis, Retina, Lens. 


\section{Introduction}

The optic vesicle, which is derived from the diencephalon, induces the formation of the lens from the overlying surface ectoderm. Not only the formation of the lens is induced, but also the direction of fibre growth, orientation of suture lines and its size is governed by the optic cup with which it is associated. The transparency of this structure is due to a highly ordered state of its fibres and the Extracellular Matrix (ECM) [1, 2].

Ontogenetically, the retina is a part of the brain. The optic vesicle develops into the optic cup that differentiates into an outer pigment epithelium and an inner retinal layer [3]. The neural retina is a complex substructure. In fact, is an outpost of the central nervous system [4]. Like the lens, the embryonic neural retina depends on the presence of the ECM molecules [5, 6]. The ECM, generally, is composed of two major classes of macromolecules: proteoglycans and fibrous proteins [7]. Type IV collagen and laminin-1 are the main fibrous proteins. Both molecules are expressed at embryonic stage day 4.5 (E4.5) in mice [8]. ECM is important for the organization of cells into tissues, it is also considered a substrate for cell attachment and adhesion, a pathway for cell movement and migration, neural guidance and maintenance of differentiated functions $[9,10]$. The ECM is very important in embryonic development. Changes in its molecules and/or genes are causal of cardiovascular, skin, renal and ocular diseases [11]. Deletion of laminin subunits, for example, leads to early death [12]. In the lens, the ECM is bound to its capsule, whilst the cells form the syncytium with the interlocking cellular processes [13]. Type IV collagen and laminin-1 of the capsule appear to be produced in the superficial lens fibres, both anterior and posterior, and in lens epithelial cells $[14,15]$.

On the other hand, in the retina, both molecules are predominantly found in the basement membrane as well as in the non-basement membrane (interstitial matrix) that is in general located between the retinal pigment epithelium and the inner limiting membrane $[16,17]$. Folic Acid (FA) is a vitamin vital for tissues development of the nervous system and specific craniofacial structures $[18,19]$ like the eye. Several studies have demonstrated the importance of FA in the nervous system specially to avoid severe disorders [20, 21]. In a previous work [22] the authors have found variations in the expression of collagen IV and laminin- 1 in the lens of mouse embryos whose mothers were subjected to FA-deficient diets, causing overexpression in some areas of the lens and underexpression in others. In the present work it is studied if alterations also take place in the texture of certain ocular tissues, especially, lens and retina. The objective is to know if in addition to producing variations in 
the concentration of collagen IV and laminin-1 changes occur in the organization of the fibres/cells of the mentioned tissues.

In order to assess changes in tissues related to deficiency of FA we carried out statistical texture analysis of the lens and retina by means of image processing techniques using Matlab. For this purpose, to assess the effect of the FA-deficiency on some eye tissues, grain size parameter is measured, and its horizontal and vertical dimensions are evaluated. The grain size is one important parameter, generally is used in speckle images to characterize the surface roughness and smoothness. It is defined as the width of the autocorrelation of tissue images [23]. In our work, we use it to determinate if there are significant changes in retinal and lens tissues that normally cannot be detected in microscopy images.

\section{Materials and methods}

\subsection{Animals and Diets}

For the present work, histological samples were taken from mouse embryos whose mothers have been subjected to diets with FA deficiency during different duration. The characteristics of the animals and diets are detailed below.

Animals: Mice were 8-week old C57/BL/6J (Harlan Laboratories, Barcelona, Spain). All mice were maintained in a $12 \mathrm{~h}$ light dark cycle at $22 \pm 2{ }^{\circ} \mathrm{C}$ and were given food and water. Diet: Female mice were divided into three groups according to the diet:

Nine female mice fed a control diet (SAFE A04 / A03 Harlan) with 2 mg FA/kg diet [24] (Control group). For studying the effects of FA diet on the texture of the retina and lens, females fed a FA-free diet with $0 \mathrm{mg} / \mathrm{kg}$ diet $(\mathrm{FAD})+1 \%$ succinylsulfathiazole (to block the synthesis of FA) for two weeks (D2 group) or eight weeks (D8 group), nine females in each group. Both diets were purchased from Harlan Laboratories, Inc., Indianapolis, Ind., USA. Immunohistochemistry:

Female mice were crossed with males fed control diet. E14.5 days later, the embryos were removed by cesarean section (equivalent to $\sim 7$ weeks of human gestation) [25], washed in $1 \%$ phosphate-buffered saline (PBS) and fixed in 4\% paraformaldehyde for 48 hours. After fixation, the embryos were then dehydrated in alcohol over several stages of increasing concentrations, and to end embedded in paraffin. Sectioning of paraffin-embedded heads is performed with a microtome (Leica Jung RN 2035) at a thickness of about $5 \mu \mathrm{m}$ and finally placed on slides. For our study an embryo was taken per mother at random. From all its slides, those corresponding to the central cut of the eye were selected for the immunohistochemical staining. 
Then, the selected slides were dried in an oven at $37^{\circ} \mathrm{C}$ for $12 \mathrm{~h}$. Before beginning the immunohistochemical techniques described below, paraffin sections have been dewaxed and rehydrated. Sections were then incubated in a solution of BSA (Bovine Serum Albumin), $1 \%$ in TRIS Buffer for 10 minutes in a dark and humid chamber. For immunohistochemistry, the polyclonal antibodies used in this experiment, and their source, outlined below: Epitope was unmasked in $5 \mu \mathrm{m}$ thick sections using a $0.2 \%$ solution of pepsin (Sigma-Aldrich) in $\mathrm{HCl}$ $0.1 \mathrm{~N}$ (for anti-collagen IV) or 1mM EDTA (Sigma-Aldrich) (for anti-laminin-1). Sections were immunolabeled for 2 hours at $37^{\circ}$ with either 1:200 polyclonal rabbit IgG anti-human type IV collagen (ICN Biomedical Inc., Aurora, OH) or 1:200 polyclonal rabbit IgG antimouse laminin (Sigma-Aldrich). Labelling was developed using the Rabbit/Mouse EnVision ${ }^{\mathrm{TM}}$ Peroxidase System, a peroxidase-conjugated dextran polymer (Dako Corp., Carpinteria, CA), and 3,30-diaminobenzidine (DAB kit) as the chromogen (Dako Corp.) [26]. All images were detected using Leica DMRB microscope and subsequently photographed using a Leica DFC 320 digital camera with x10 magnification. The sample (n) were subdivided according to the diet: control group $(\mathrm{n}=9)$, D2 group $(\mathrm{n}=9)$ with FAD diet for 2 weeks, and finally D8 ( $\mathrm{n}=9)$ with FAD diet for 8 weeks.

Image processing and Statistical Analysis of the immunohistochemical data was performed using Matlab®.

\subsection{Image processing analysis methods}

As stated above, nine slides of each group labelled with anti-type IV collagen and antilaminin-1 proteins were used to study changes in texture that occurred in the lens and retina when there was a deficit of FA; i.e., analysing how FA deficiency affects both proteins. For texture analysis, we used three groups of samples for both collagen IV and laminin-1 images which are: the control, D2, and D8 groups.

All images used were grayscale normalized (see Fig. 1B) to have the same range of signal; this was done to investigate the texture of the tissues. Figure 1A represents the local zone in the lens and retina studied (ROI-Region of Interest). All anti-type IV collagen group images were sized 293x308; whereas the anti-laminin-1 group image size was 172x245. Figure 1B represents one of these selected areas and Fig. 1C represents the results of applying canny edge detector to the selected area. Canny edge detection is an image processing tool used to detect the structure of the edge of an image; segmentation based on canny edge detection is fast and does not require high computational power [27, 28]. This type of tool is normally 
used to study the structure of tissue texture given by fibres, grains, etc. [29, 30]. All images were fully aligned before applying image processing.

For each sample group (Control, D2, and D8) auto-correlation was performed on each image individually after removing the mean image value. For this purpose, autocorrelation function of images was calculated with Matlab software applied to the type of tissue images of Fig. 1B. It is possible to measure the main grain size for each group and zone as the width of the autocorrelation function. In our case we took the half-height width in the horizontal and vertical directions and the final value of grain size was taken as the geometric mean value of both directions. Finally, we considered the mean value of the autocorrelation function width as a correlation representative of the whole group. The correlation between image pixels provides information about the shape and average grain size in the digital image. In summary, the correlation gave a picture of both staining size: type IV collagen and laminin-1.

On the other hand, Fourier Transform (FT) is an important tool in image processing often used to obtain information about the geometric structure of the image in the frequency domain. In fact, FT has become indispensable in many scientific areas such as biomedicine [31]. For this analysis we used the same images of the previous correlation analysis but now with a prior canny edge treatment (see Fig. 1C) for finding contours present in images. For each sample group, FT was performed on each image individually. In Matlab we applied functions, fft2 (to return the two-dimensional FT of the image) and fftshift (shifts zerofrequency component to centre of spectrum). In order to increase the accuracy, the fft 2 is calculated with 1000x1000 points, so each original image is padded with zeros. In this way, the $(0,0)$ point in Fourier frequency plane is located in $(500,500)$ point in the FT. As an example, the image of vertical lines three pixels spaced is shown in Fig. 1D. Its FT is shown in Fig. 1E. It consists of two frequency points in the horizontal axis showing that the image consists of vertical lines of high frequency (the points are far from the $(0,0))$. Then, the spacing and orientation of the lines of the image of the canny edges can be studied by means of the image of its FT.

In our case, the set of 9 FT-images for each group was averaged and the final image was taken as representative of the whole group. Then, FT allowed us to analyse the distribution of protein staining for type IV collagen and laminin-1, and its fibres/cells orientation.

\subsection{Statistical analysis:}

The bootstrap is a resampling method for statistical inference, generally, used for estimating the distributions of statistics based on independent observations. This method draws repeated 
samples that has the same size from an estimate of the population a number of times [32]. It is usually used when the base distribution of the data is unknown. It provides an estimate of the probability distribution of various parameters such as the mean, median, standard deviation, etc. In our case, these methods have been used to estimate the distribution and confidence intervals of the mean, standard deviation, skewness and kurtosis of the grain size of the tissues. A total of 100 bootstrap replicates were used to compute samples of the previous parameters. This sample size is usually sufficient to give adequate confidence intervals [33]. We then used the Kruskal-Wallis test, a non-parametric ANOVA, to test when the distributions of the mean, standard deviation, skewness and kurtosis are different between the control and the deficient groups, D2 and D8, at a level of significance $p<0.5$. All the analyses were performed in Matlab.

\section{Results:}

\subsection{Results for lens texture}

\section{Grain size}

In this section the results of texture images processing to the lens images are exposed. The first results are shown in Figure 2. In the case of type IV collagen, (see Figs. 2(A, a), 2(B, b), and $2(\mathrm{C}, \mathrm{c}))$, we found that grain size became larger with increasing FA deficiency. Especially in D8 where we clearly see that grain size was larger in height and width. However, the situation with laminin-1 images was completely different, (see Figs. 2(D, d), 2(E, e), and 2(F, f)). We found that grain size grew smaller with increasing deficit. Grain size started to shrink in D2 and it was much smaller in D8 compared to control group.

Figs. 3 and 4 show the results of the statistical analysis performed for the lens grain size data. To do that, samples of 100 data have been simulated with the bootstrap method. We have tested with bootstrap samples of different sizes and the number 100 gave stable results without increasing the number of samples too much respect to the original [33]. With this method, 100 samples of the mean values, standard deviation, skewness and kurtosis have been obtained from the nine grain size values for the control, D2 and D8 groups. After this, a nonparametric Kruskal-Wallis contrast is applied to compare the equality of the distribution of the previous parameters between the control group and the FA-deficient groups. The results are shown in the form of Tukey's box plot, described by Tukey in 1977, that uses five values from the set of data: the extremes represent the minimum and maximum observations, the upper and lower hinges (quartiles), and the median (line through the box) [34]. In our 
study we utilize this box plot for summarizing graphically the frequency distribution of our variables of interest. Figure 3 shows the results for lenses labelled with anti-collagen IV and figure 4 for lenses labelled with anti-laminin1. Each figure contains three box plots of the spatial distribution of control, D2, and D8 groups. In case of anti-collagen IV, the differences are statistical significant $(\mathrm{p}<0.05)$ between control-D2 and control-D8 in all parameters (mean, standard deviation, skewness and kurtosis). This shows that the probability distribution of grain size is different between control group and D2 and D8 groups. In case of laminin-1 all the parameters are again different except kurtosis. These results suggest that there are changes in the size of the lens grain with only two weeks of FA deficit. On average, the size of the grain increases in tissues labelled with anti-collagen IV and decreases in the case of anti-laminin-1 when the FA-deficiency time increases. The median of the probability distribution of each statistical estimator (mean, standard deviation, skewness and kurtosis) are summarized in Table 1. These median values are those marked in the graphs with the central horizontal line of the boxes of the Tukey box graphics.

\section{Fibre orientation:}

Figure 5 shows the results for the fibre orientation analysis using FT. In each of images, it can be observed that the spectrum has its principal values in a circular shape showing that the edge fibres detected by the canny-edge method mainly change their orientations and not the distance between them. The anti-collagen IV images (see Figs. 5(A, a), 5(B, b), and 5(C, c)) show that dispersion was less in D2 and D8; this was also accompanied by a small change in the orientation of D2 and D8 groups. However, images labelled with anti-laminin-1 (see Figs. 5(D, d), 5(E, e), and 5(F, f) ) show exactly the opposite: the dispersion of the orientation increases slightly in D2 and D8; in this case the orientation of the fibres does not change either.

\subsection{Results for retinal texture}

\section{Grain size}

Retina texture analysis followed the same procedure as was performed for the lens. Images labelled with type IV collagen and laminin-1 showed that severe FA-deficiency produced an increase in grain size, see Fig. 6.

As in the case of the lens, for each individual the geometric mean between horizontal and vertical grain size and its statistical values in each group are analysed. To do this, bootstrap 
samples of size 100 samples are taken to generate distributions of the mean value, standard deviation, skewness and kurtosis of grain size data for each group (control, D2, D8). The level of statistical significance of the differences between the bootstrap distributions of these parameters between the control group and D2, D8, respectively, are analysed again with the nonparametric Kruskal-Wallis test. The results, in the form of Tukey box plot are shown in Figs. 7 and 8. The differences are all significant $(\mathrm{p}<0.05)$ except in the case of the kurtosis of the grain size of the retinas marked with anti-collagen IV. In this case, the grain size increases in mean value respect to control for retinas labelled with both proteins (collagen IV and laminin-1). The median of the probability distribution of each statistical estimator (mean, standard deviation, skewness and kurtosis) are summarized in Table 2. These median values are those marked in the graphs with the central horizontal line of the boxes of the Tukey box graphics.

\section{Fibre orientation:}

Like the lens, FA deficiency produced greater dispersion in the orientation of fibres for the two analysed molecules (collagen IV and laminin-1), but not relevant change in the mean value of orientation was observed among the three groups (see Figs. 9).

\section{Discussion}

Few organs of the body have as extensive an ECM as the eye [17]. Several interstitial ECM components such as type IV collagen and laminin-1 have been identified in the lens and retina of mice in E14.5 [5, 6, 35-37]. Type IV collagen forms an integrated network within the laminin-1 scaffold providing strength and stability [15]. These proteins are important as structural components of the native basement membranes and subsequently as matrices supporting cellular adhesion [38]. It is not surprising that any disruption in type IV collagen or laminin-1 would result in changes or alterations in tissue morphology.

The image processing morphological analysis showed that FA deficiency directly altered type IV collagen and laminin-1 in the lens and retina. In this study, we successfully observed for the first time that FA deficiency produced a remarkable change in the retinal and lens tissues. Therefore, the collagen and laminin-1 structure as revealed by our technique change with FA deficiency. 
In the case of type IV collagen (Figs. 2(A,a), 2(B,b), and 2(C,c)), the tissue morphology in the control lenses observed in this study had a smooth and homogeneous texture. In comparison, FA-deficient lenses tissue was coarser, granulated and more irregular. In the case of laminin-1 (Figs. 2(D,d), 2(E,e), and 2(F,f)) the tissue behaves contrary to the collagen case, i.e. tissue was rough and granulated in the control group but it became uniform, homogeneous and smooth in both deficient groups. Our previous study [22] also found that FA alters spatial pattern of expression of collagen IV and laminin-1 inside the lens. In that research, we have seen that due to the FA deficiency there is an overexpression of collagen IV inside the lens; however, in laminin-1 FA deficiency has opposite effect, an underexpression. These results agree with the current results where we observed an increase in grain size in the case of collagen IV and its decrease in the case of laminin-1. However, in the present study, the morphological changes were accompanied in the case of type IV collagen with a decrease in the dispersion and deviation in orientation of fibres; in control and D2 individuals the collagen was oriented vertically, but in D8 the orientation had an oblique inclination and collagen was irregularly arranged (Figs. 5(A,a), 5(B,b), and $5(\mathrm{C}, \mathrm{c}))$. In contrast, sections labelled with laminin-1 presented an insignificant change in the orientation (Figs. 5(D,d), 5(E,e), and 5(F,f)).

Epithelial cells attach preferentially to type IV collagen and this attachment is slow [39]. Laminin-1 is considered the attachment factor for these cells [40]. In the presence of laminin1, epithelial cells attach rapidly to type IV collagen. After that, the cells bind to the laminintype IV collagen complex [41]. Perhaps FA in some way alters this attachment between type IV collagen and laminin-1 and consequently lens texture changes; which can result in lens abnormalities, including lenticonus, abnormal lens fibre growth, cataract, etc. Some of these abnormalities have been found in diseases such as Alport Syndrome because of changes in type IV collagen expression [42, 43].

Nevertheless, in control retinas labelled with anti-type IV collagen, the tissue was rough and granulated; it changed to uniform and homogenous in D2 and D8 respectively (Figs. 6(A,a), $6(\mathrm{~B}, \mathrm{~b})$, and $6(\mathrm{C}, \mathrm{c}))$. The orientation was the same in all groups, but dispersion changed slightly in deficient groups (Figs. 9(A,a), 9(B,b), and 9(C,c)). Similarly, we analysed all retinal tissues labelled with anti-laminin-1. In this case, and as expected, the results were different. In this case retinal tissue morphology in control individuals was very homogenous and uniform. However, the morphology changed in deficient groups and turned granulated (Figs. 6(D,d), 6(E,e), and 6(F,f)). Regarding the orientation, the change was minimal but more dispersion was found in the mice with deficit (Figs. 9(D,d), 9(E,e), and 9(F,f)). In 
summary, the changes were observed in tissues immunolabeled with anti-type IV collagen and anti-laminin-1; this means that at the retinal level, the FA deficit changed both molecules. Type IV collagen and laminin play important role in neural retina growth, both are expressed in its interstitial matrix during development $[6,44]$. It has been shown that alterations in these proteins produce disruption to retinal morphology and physiology [45, 46]. Loss of lamininmediated signalling in the retina results in retinal dysplasia and may lead to visual impairment $[47,48]$.

\section{Conclusions}

Texture and image processing techniques such as Canny edge detection, Fourier transform, and auto-correlation were used to analyse and characterize changes in biological tissues. Using these approaches, it was possible to distinguish between normal and abnormal tissues by choosing a region of interest (ROI) and comparing it to control regions. In conclusion, these methods have potential applicability in microscopic sections for evaluating morphological changes in tissues. Using these digital image processing tools may become another instrument for assessing and characterizing biomedical tissues. There is no doubt that these tools are easier and faster methods for obtaining a preliminary analysis of biological tissues.

In summary, the severe ocular alterations observed in our FA deficient mice embryos in texture analysis revealed the important role of ECM molecules during embryonic development. It is evident that FA plays a significant role in ECM protein synthesis and FA deficiency contributes to many kinds of ocular disorders. Changes introduced by FA deficiency were visible in the short-term (two weeks) and clearly evident in the long term (eight weeks) in both grain size and orientation of fibre structures in the tissues analysed (lens and retina). In our knowledge this is the first work devoted to study the effect of FA deficit in the texture of eye tissues. 
Disclosures: No conflicts of interest, financial or otherwise, are declared by the authors.

Funding: This study was supported by grants to the Complutense Research Group 920202 from the Spanish Ministry of Health (PI06/0184 and PS09/01762). Likewise, it was supported by grants from the Spanish Ministry of Economy and competitiveness (TEC201340442). Finally, this article was done with a pre-doctoral fellowship awarded by Complutense University of Madrid and Santander bank (reference number CT27/16 - CT28/16).

Acknowledgments: The authors thank Alda Serrano Javier from Optics Department for reviewing the draft of the paper and for his helpful suggestions and comments on this manuscript. The authors would also like to acknowledge Dolores Arroyo and Alicia Cerro from the Anatomy and Human Embryology Department for their excellent technical assistance.

\section{Compliance with ethical standards:}

Ethical approval: Animal experiments: All applicable international, national, and/or institutional guidelines for the care and use of animals were followed. All procedures performed in studies involving animals were in accordance with the ethical standards of the Animal Experimentation Committee of the Universidad Complutense of Madrid (UCM). The mice were maintained at animal house of the School of Medicine at the UCM. 
1. Smelser GK (1965) Embryology and morphology of the lens. Investigative Ophthalmology \& Visual Science 4: 398-410

2. Rao PV, Maddala R (2006) The role of the lens actin cytoskeleton in fiber cell elongation and differentiation. Seminars in Cell \& Developmental Biology 17: 698-711 DOI 10.1016/j.semcdb.2006.11.011

3. Pei Y, Rhodin J (1970) The prenatal development of the mouse eye. The Anatomical Record 168: $105-125$

4. Smith RS, John SW, Nishina PM, Sundberg JP (2001) Systematic evaluation of the mouse eye: anatomy, pathology, and biomethods. CRC press, Boca Ratan.

5. Sarthy PV, Fu M (1990) Localization of laminin B1 mRNA in retinal ganglion cells by in situ hybridization. The Journal of cell biology 110: 2099-2108

6. Taylor L, Arnér K, Engelsberg K, Ghosh F (2015) Scaffolding the retina: The interstitial extracellular matrix during rat retinal development. International Journal of Developmental Neuroscience 42: 46-58

7. Schaefer L, Schaefer RM (2010) Proteoglycans: from structural compounds to signaling molecules. Cell and Tissue Research 339: 237-246 DOI 10.1007/s00441-009-0821-y

8. LeBleu VS, MacDonald B, Kalluri R (2007) Structure and function of basement membranes. Experimental biology and medicine 232: 1121-1129

9. Frantz C, Stewart KM, Weaver VM (2010) The extracellular matrix at a glance. J Cell Sci 123: 4195-4200

10. Rozario T, DeSimone DW (2010) The extracellular matrix in development and morphogenesis: a dynamic view. Developmental biology 341: 126-140

11. Bateman JF, Boot-Handford RP, Lamandé SR (2009) Genetic diseases of connective tissues: cellular and extracellular effects of ECM mutations. Nature Reviews Genetics 10: 173-183

12. Hirrlinger PG, Pannicke T, Winkler U, Claudepierre T, Varshney S, Schulze C, Reichenbach A, Brunken WJ, Hirrlinger J (2011) Genetic deletion of laminin isoforms $\beta 2$ and $\gamma 3$ induces a reduction in Kir4. 1 and aquaporin-4 expression and function in the retina. PLoS One 6: e16106

13. Forrester JV, Dick AD, McMenamin PG, Roberts F, Pearlman E (2015) The eye: basic sciences in practice. Elsevier Health Sciences, Edimburgh.

14. Young RW, Ocumpaugh DE (1966) Autoradiographic studies on the growth and development of the lens capsule in the rat. Invest Ophthalmol 5: 583-589

15. Danysh BP, Duncan MK (2009) The lens capsule. Experimental eye research 88: 151-164

16. Reinhard J, Joachim SC, Faissner A (2015) Extracellular matrix remodeling during retinal development. Experimental eye research 133: 132-140

17. Hausman RE (2007) Ocular extracellular matrices in development. Progress in retinal and eye research 26: 162-188

18. Tang LS, Santillano DR, Wlodarczyk BJ, Miranda RC, Finnell RH (2005) Role of Folbp1 in the regional regulation of apoptosis and cell proliferation in the developing neural tube and craniofaciesAmerican Journal of Medical Genetics Part C: Seminars in Medical Genetics. Wiley Online Library, pp. 48-58.

19. Maddox DM, Manlapat A, Roon P, Prasad P, Ganapathy V, Smith SB (2003) Reduced-folate carrier (RFC) is expressed in placenta and yolk sac, as well as in cells of the developing forebrain, hindbrain, neural tube, craniofacial region, eye, limb buds and heart. BMC developmental biology 3: 6

20. Zhao Y, Huang G, Chen S, Gou Y, Dong Z, Zhang X (2016) Folic acid deficiency increases brain cell injury via autophagy enhancement after focal cerebral ischemia. The Journal of nutritional biochemistry 38: 41-49 
21. Kao T-T, Chu C-Y, Lee G-H, Hsiao T-H, Cheng N-W, Chang N-S, Chen B-H, Fu T-F (2014) Folate deficiency-induced oxidative stress contributes to neuropathy in young and aged zebrafishImplication in neural tube defects and Alzheimer's diseases. Neurobiology of disease 71:

234-244

22. Sijilmassi O, López Alonso JM, Barrio Asensio MC, Del Río Sevilla A (2018) Collagen IV and laminin-1 expression in embryonic mouse lens using principal components analysis technique. Journal of Microscopy, doi:10.1111/jmi.12709

23. Nassif R, Pellen F, Magne C, Le Jeune B, Le Brun G, Abboud M (2012) Laser speckle dynamic for monitoring fruits maturationSpeckle 2012: V International Conference on Speckle Metrology. International Society for Optics and Photonics, pp. $84131 \mathrm{G}$.

24. COUNCIL NR COMMITTEE ON ANIMAL NUTRITION. SUBCOMMITTEE ON POULTRY NUTRITION (1995) Nutrient Requirements of Poultry. Washington: National Academy Press.

25. Otis EM, Brent R (1954) Equivalent ages in mouse and human embryos. The Anatomical record 120: 33-63

26. Martínez-Sanz E, Del Río A, Barrio C, Murillo J, Maldonado E, Garcillán B, Amorós M, Fuerte T, Fernández Á, Trinidad E (2008) Alteration of medial-edge epithelium cell adhesion in two Tgf- $\beta 3$ null mouse strains. Differentiation 76: 417-430

27. Gonzalez RC, Woods RE, Eddins SL, (2004) Digital image processing using MATLAB. Pearson Prentice Hall, New Jersey.

28. Canny J (1986) A computational approach to edge detection. IEEE Transactions on pattern analysis and machine intelligence: $679-698$

29. Hwang J, Kim M, Kim S, Lee J (2013) Quantifying morphological parameters of the terminal branching units in a mouse lung by phase contrast synchrotron radiation computed tomography. PLoS One 8: e63552

30. Shim D-S, Chang S (2008) Sub-pixel retinal vessel tracking and measurement using modified canny edge detection method. Journal of Imaging Science and Technology 52: 20505-2050120505-20506

31. Li Y, Chen WR, Zhang Y, Qian W, Liu H (2002) Comparison of analog and digital Fourier transforms in medical image analysis. Journal of biomedical optics 7: 255-261

32. Efron B, Tibshirani RJ (1993) An Introduction to the Bootstrap: Monographs on Statistics and Applied Probability, Vol. 57. New York and London: Chapman and Hall/CRC

33. Goodhue DL, Lewis W, Thompson R (2012) Does PLS have advantages for small sample size or non-normal data? Mis Quarterly: 981-1001

34. Tukey JW (1977) Exploratory data analysis. Reading, Masschusetts: Addison-Wesley.

35. Sarthy V (1993) Collagen IV mRNA expression during development of the mouse retina: an in situ hybridization study. Investigative ophthalmology \& visual science 34: 145-152

36. Parmigiani C, McAvoy J (1984) Localisation of laminin and fibronectin during rat lens morphogenesis. Differentiation 28: 53-61

37. Rafighdoost $H$, Jalali M, Nikravesh MR (2013) Evalauation of Laminin Expression during Mouse Lens Development. Anatomical Sciences Journal 10: 1-6

38. Olivero DK, Furcht LT (1993) TYPE-IV COLLAGEN, LAMININ, AND FIBRONECTIN PROMOTE THE ADHESION AND MIGRATION OF RABBIT LENS EPITHELIAL-CELLS IN-VITRO. Investigative Ophthalmology \& Visual Science 34: 2825-2834

39. Hughes RC, Mills G, Courtois Y (1979) ROLE OF FIBRONECTIN IN THE ADHESIVENESS OF BOVINE LENS EPITHELIAL-CELLS. Biologie Cellulaire 36: 321-329

40. Terranova VP, Rohrbach DH, Martin GR (1980) Role of laminin in the attachment of PAM 212 (epithelial) cells to basement membrane collagen. Cell 22: 719-726

41. Kleinman HK, Klebe RJ, Martin GR (1981) ROLE OF COLLAGENOUS MATRICES IN THE ADHESION AND GROWTH OF CELLS. Journal of Cell Biology 88: 473-485 DOI 10.1083/jcb.88.3.473 
42. Savige J, Sheth S, Leys A, Nicholson A, Mack HG, Colville D (2015) Ocular Features in Alport Syndrome: Pathogenesis and Clinical Significance. Clinical Journal of the American Society of Nephrology 10: 703-709 DOI 10.2215/cjn.10581014

43. Ohkubo S, Takeda H, Higashide T, Ito M, Sakurai M, Shirao Y, Yanagida T, Oda Y, Sado Y (2003) Immunohistochemical and molecular genetic evidence for type IV collagen alpha 5 chain abnormality in the anterior lenticonus associated with Alport syndrome. Archives of Ophthalmology 121: 846-850 DOI 10.1001/archopht.121.6.846

44. Kuida K, Zheng TS, Na SQ, Kuan CY, Yang D, Karasuyama H, Rakic P, Flavell RA (1996) Decreased apoptosis in the brain and premature lethality in CPP32-deficient mice. Nature 384: 368-372 DOI 10.1038/384368a0

45. Libby RT, Lavallee CR, Balkema GW, Brunken WJ, Hunter DD (1999) Disruption of laminin beta 2 chain production causes alterations in morphology and function in the CNS. Journal of Neuroscience 19: 9399-9411

46. Favor J, Gloeckner CJ, Janik D, Klempt M, Neuhauser-Klaus A, Pretsch W, Schmahl W, Quintanilla-Fend L (2007) Type IV procollagen missense mutations associated with defects of the eye, vascular stability, the brain, kidney function and embryonic or postnatal viability in the mouse, Mus musculus: An extension of the Col 4 alpha 1 allelic series and the identification of the first two Col4a2 mutant Alleles. Genetics 175: 725-736 DOI 10.1534/genetics.106.064733

47. Satz JS, Philp AR, Nguyen H, Kusano H, Lee J, Turk R, Riker MJ, Hernandez J, Weiss RM, Anderson MG, Mullins RF, Moore SA, Stone EM, Campbell KP (2009) Visual Impairment in the Absence of Dystroglycan. Journal of Neuroscience 29: 13136-13146 DOI 10.1523/jneurosci.0474-09.2009

48. Pinzon-Duarte G, Daly G, Li YN, Koch M, Brunken WJ (2010) Defective Formation of the Inner Limiting Membrane in Laminin beta 2-and gamma 3-Null Mice Produces Retinal Dysplasia. Investigative Ophthalmology \& Visual Science 51: 1773-1782 DOI 10.1167/iovs.09-4645 


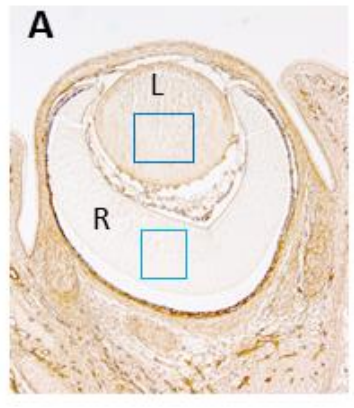

B

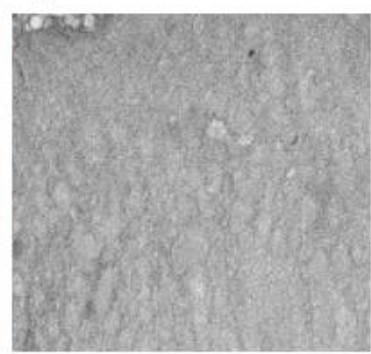

D

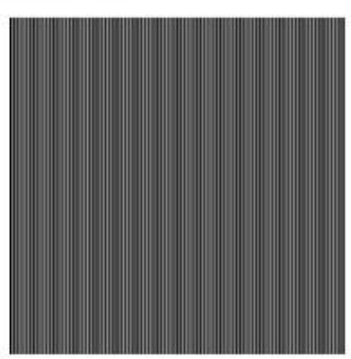

C

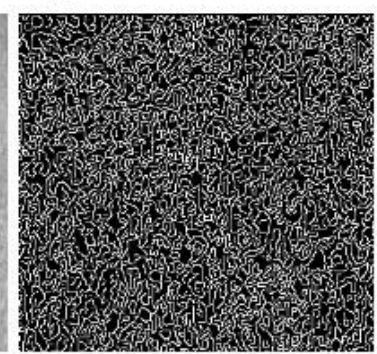

$\mathbf{E}$

Fig. 1: A) Frontal section of the eye of a control mouse labelled with anti-type IV collagen (size $5 \mu \mathrm{m})$. The squared areas are the regions analysed. L: lens. R: retina. B) Local region of the lens of a control mouse in grayscale. C) Local region of the lens of a control mouse with canny edge detector. D) Image of vertical lines spaced 3 pixels and E) its Fourie Transform Image.
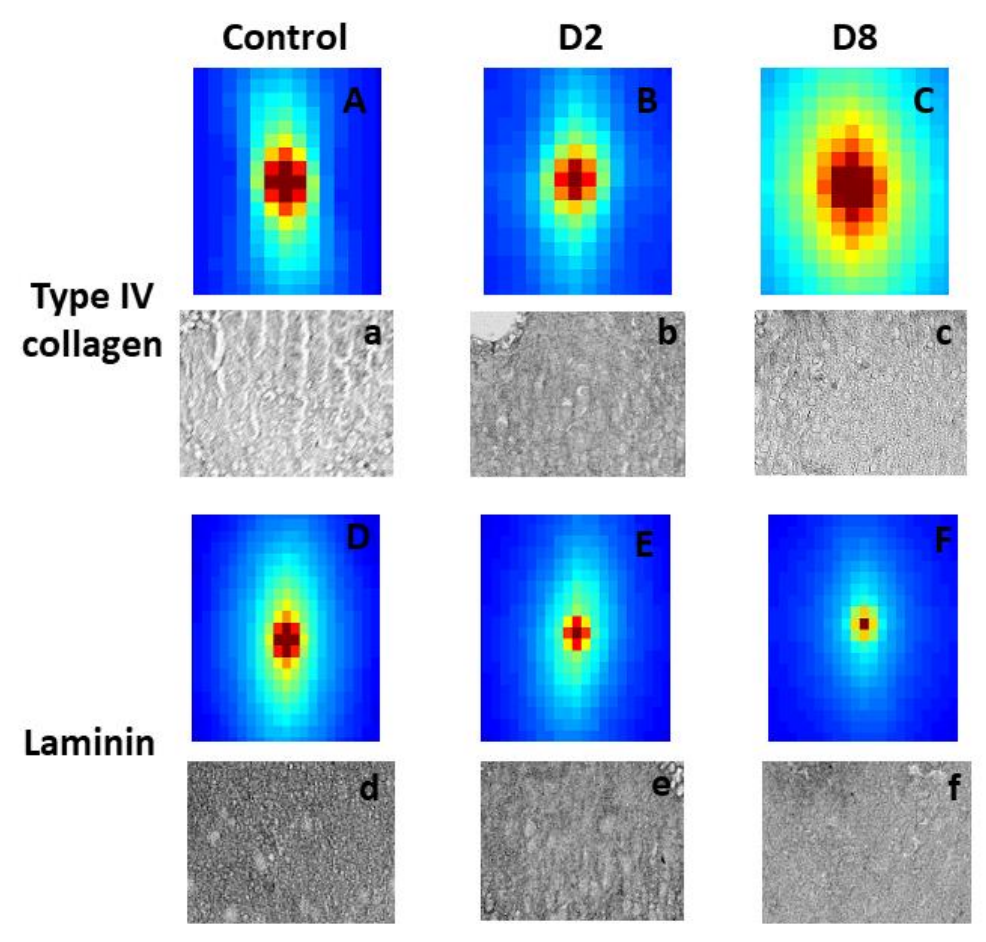

Fig. 2: Correlation of grain size obtained from lens image analysis. Figures A, B and C represent control, D2 and D8 groups labelled with anti- type IV collagen. Figures D, E and F represent control, D2 and D8 groups labelled with anti-laminin (Correlations). Figures a, b, and c correspond to control, D2, and, D8 lens tissues labelled with anti-type IV collagen. Figures $\mathrm{d}, \mathrm{e}$, and f correspond to control, D2, and D8 lens tissues labelled with anti-laminin-1. 

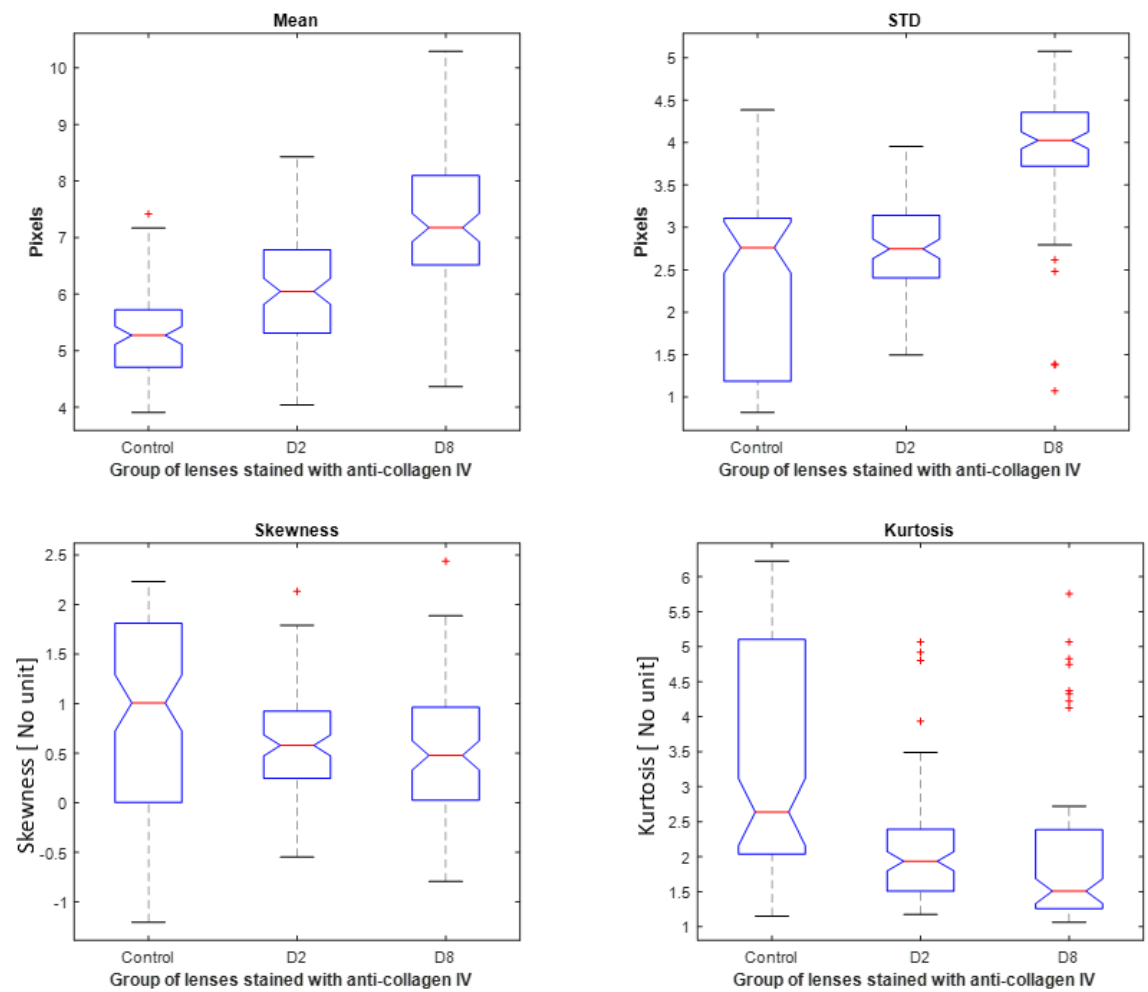

Fig. 3: Tukey box plot for the mean, standard deviation, skewness and kurtosis of the grain size of the lens tissues labelled with anti-collagen IV. All groups, for bootstrap samples of 100 data, show statistically significant difference for $\mathrm{p}<0.05$.
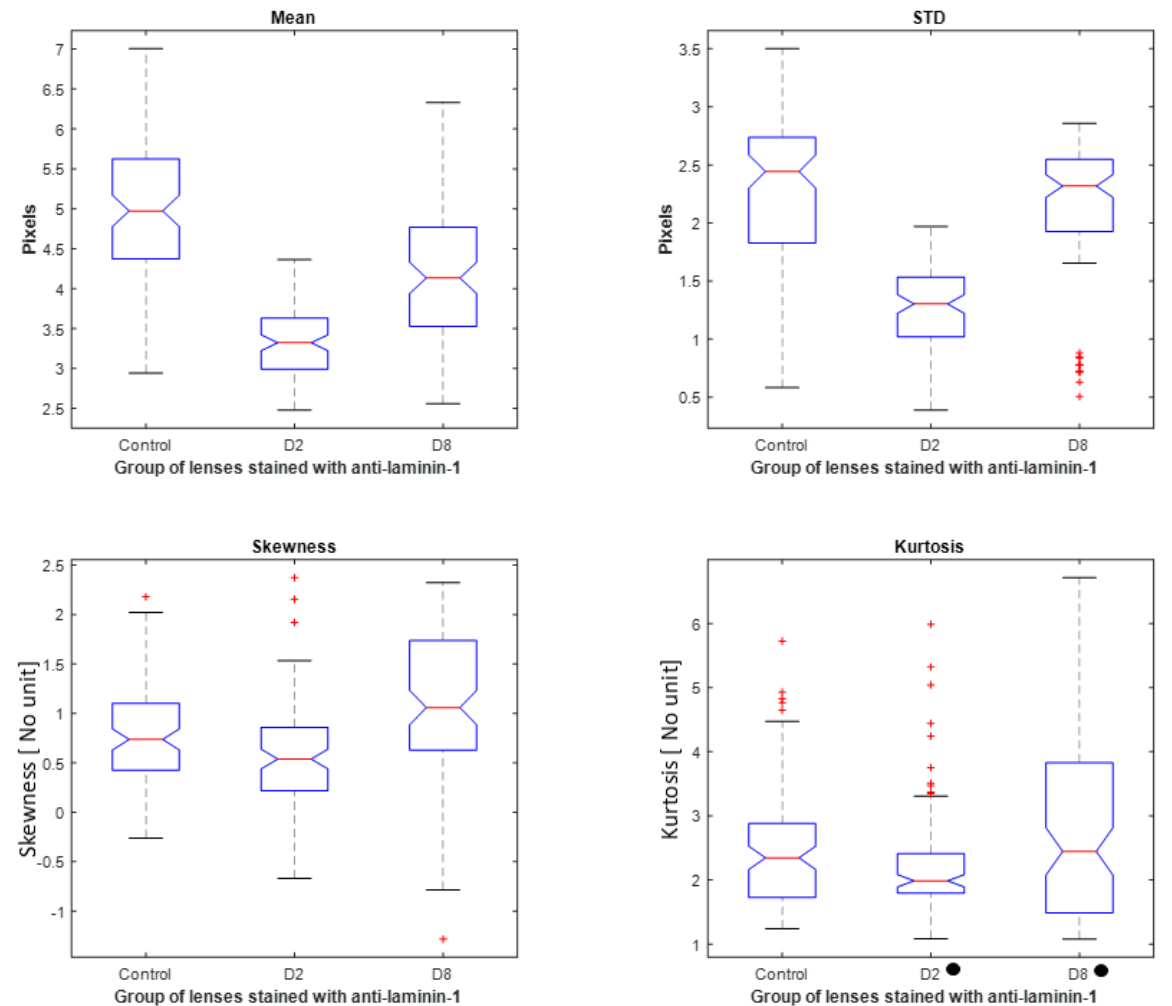

Fig. 4: Tukey box plot for the mean, standard deviation, skewness and kurtosis of the grain size of the lens tissues labelled with anti-laminin-1. All groups, for bootstrap samples of 100 data, show statistically significant difference for $p<0.05$, except kurtosis (labelled with "black points" in figure). 


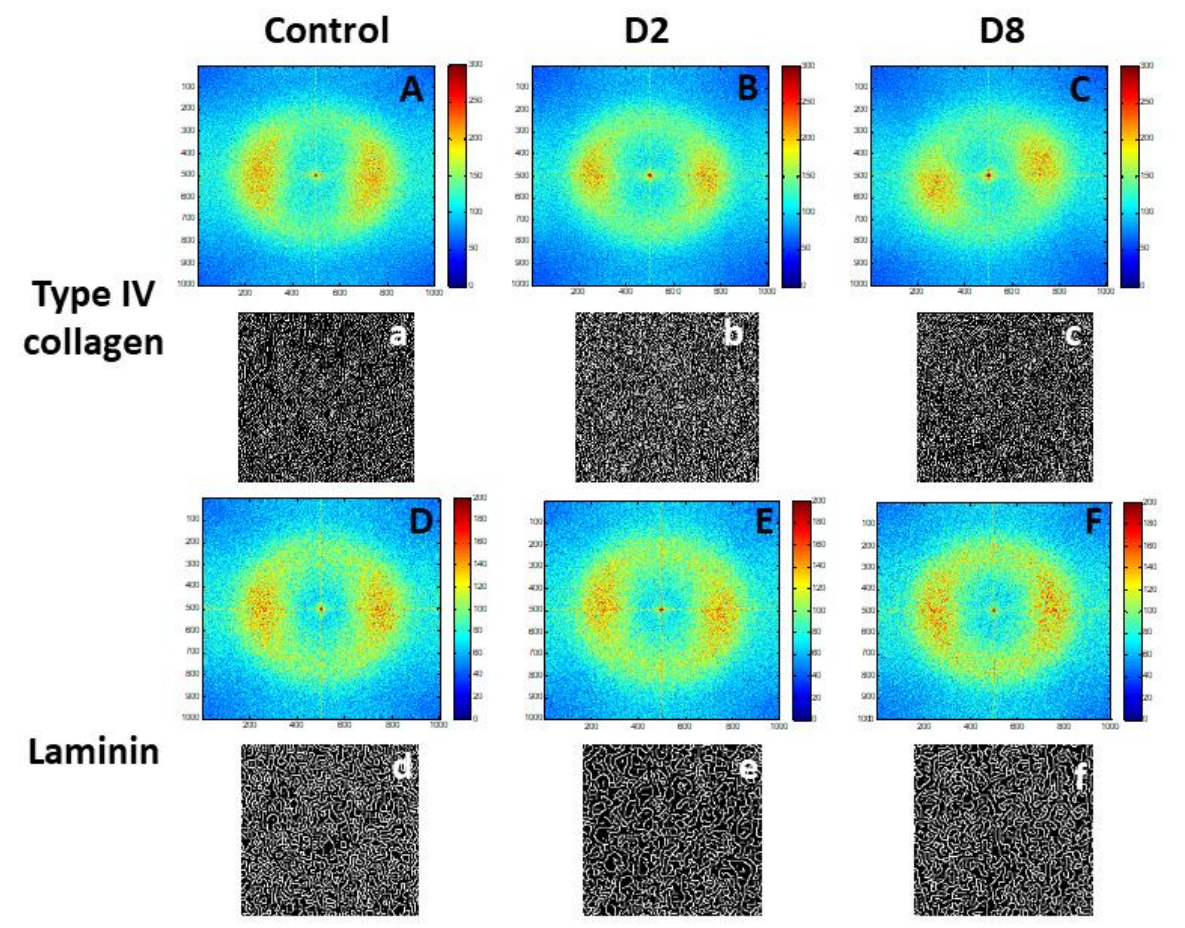

Fig. 5: Fourier Transform analysis of the lens. Figures A, B and C represent control, D2 and D8 groups marked with antitype IV collagen. Figures D, E and F represent control, D2 and D8 groups labelled with anti-laminin. Figures a, b, and c correspond to lens canny edge detection from control, D2 and D8 labelled with anti-type IV collagen. Figures d, e and f correspond to lens canny edge detection from control, D2 and D8 labelled with anti-laminin-1.

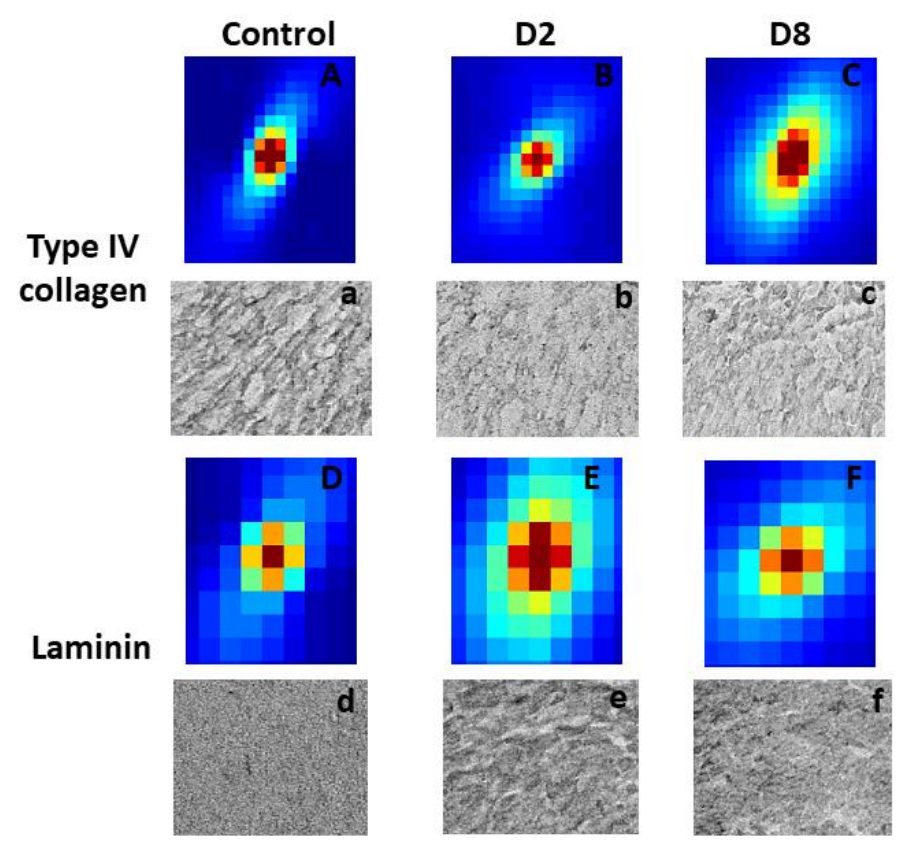

Fig. 6: Correlation of grain size obtained from retinal image analysis. Figures A, B and C represent control, D2 and D8 groups labelled with anti-type IV collagen. Figures D, E and F represent control, D2 and D8 groups labelled with antilaminin (Correlations). Figures a, b and c correspond to control, D2 and D8 retinal tissue labelled with anti- type IV collagen. Figures d, e, and f correspond to control, D2 and D8 retinal tissue labelled with anti-laminin-1. 

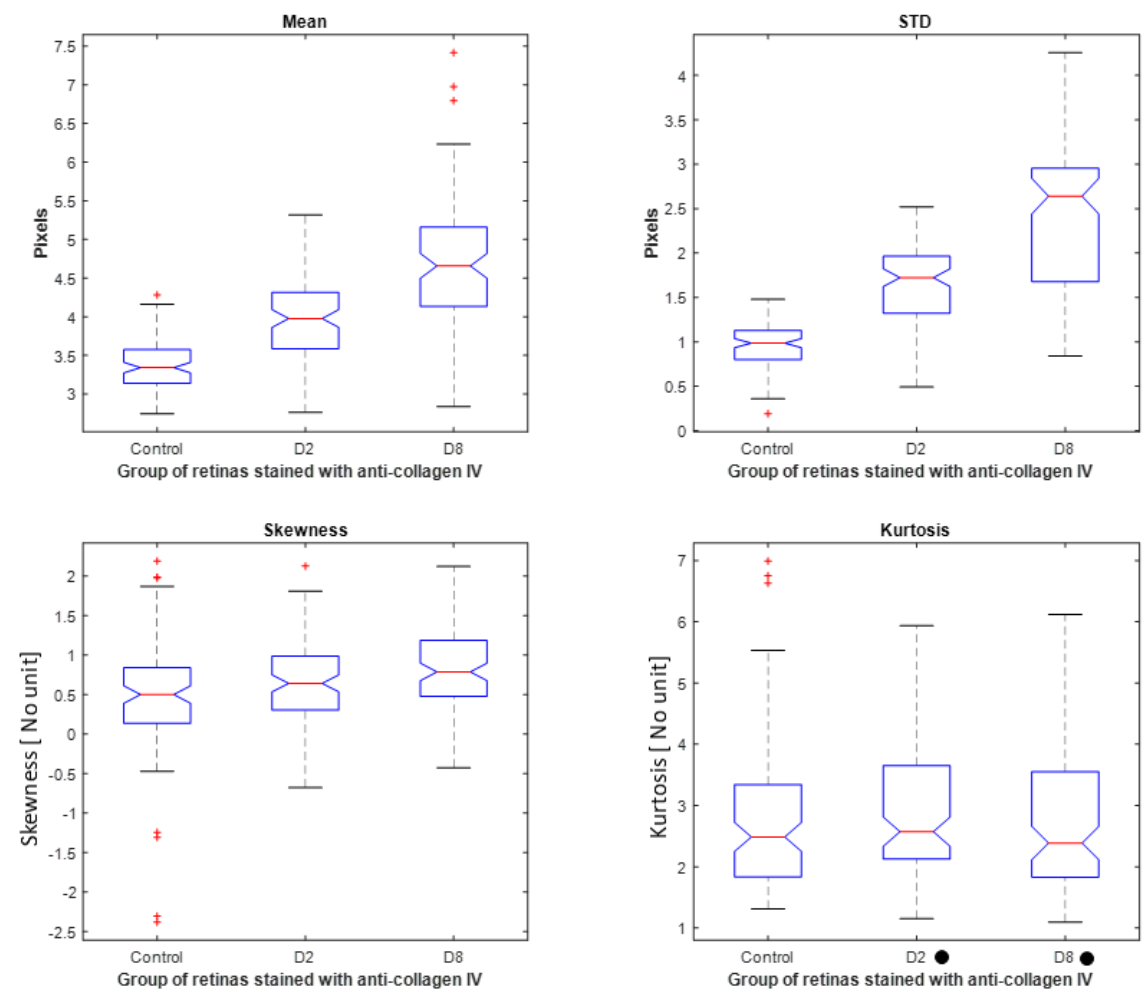

Fig. 7: Tukey box plot for the mean, standard deviation, skewness and kurtosis of the grain size of the retinas labelled with anti-collagen IV. All groups, for bootstrap samples of 100 data, show statistically significant difference for $p<0.05$, except kurtosis.
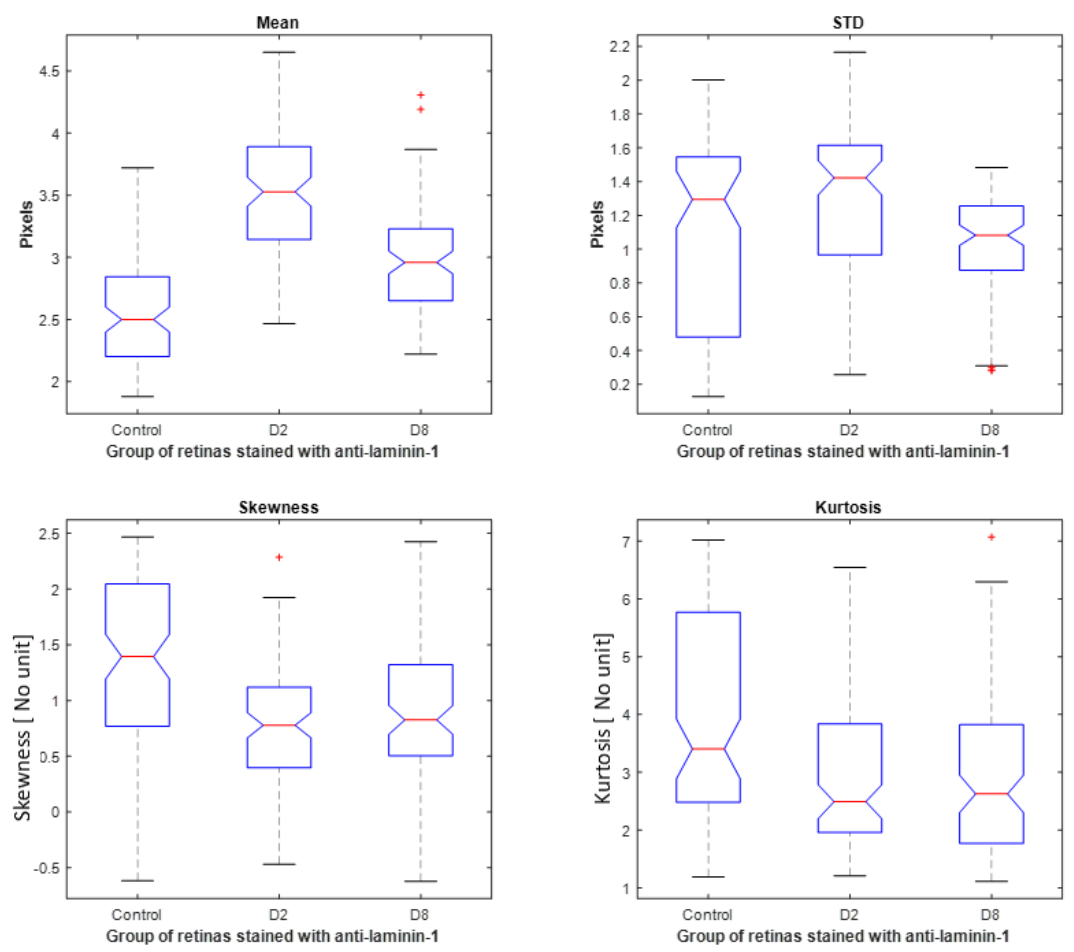

Fig. 8: Tukey box plot for the mean, standard deviation, skewness and kurtosis of the grain size of the retinas labelled with anti-laminin-1. All groups, for bootstrap samples of 100 data, show statistically significant difference for $p<0.05$. 


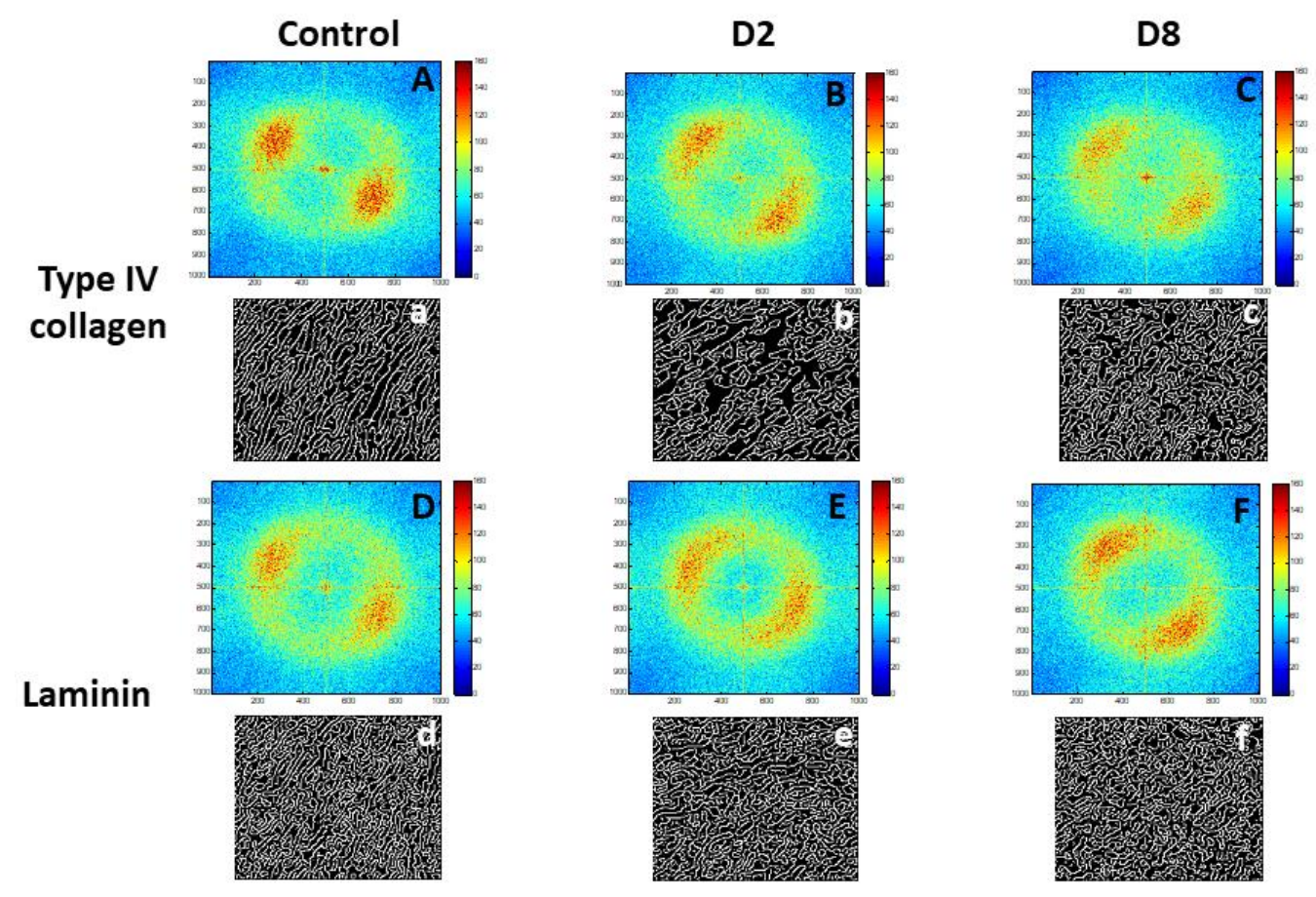

Fig. 9: Fourier Transform analysis of retinal images. Figures A, B and $C$ represent control, D2 and D8 groups marked with anti- type IV collagen. Figures D, E and F represent control, D2 and D8 groups labelled with anti-laminin. Figures a, b and c correspond to retinal canny edge detection from control, D2 and D8 labelled with anti- type IV collagen. Figures d, e and f correspond to retinal canny edge detection from control, D2 and D8 labelled with anti-laminin-1.

Table 1: Medians of statistical estimators (bootstrap) of grain size probability distribution (mean, standard deviation, skewness and kurtosis) of the lenses marked with anti-collagen IV or anti-laminin-1 from control and FA deficient groups D2 and D8. (*): not significant difference in estimator probability distribution.

\begin{tabular}{|l|c|c|c|c|c|c|c|c|c|}
\cline { 2 - 10 } \multicolumn{1}{c|}{} & \multicolumn{3}{c|}{ Mean \pm std } & \multicolumn{3}{c|}{ Mean Skewness } & \multicolumn{3}{c|}{ Mean Kurtosis } \\
\cline { 2 - 10 } \multicolumn{1}{c|}{} & Control & D2 & D8 & Control & D2 & D8 & Control & D2 & D8 \\
\hline $\begin{array}{l}\text { Lenses stained } \\
\text { with anti- } \\
\text { collagen IV }\end{array}$ & $5.3 \pm 2.8$ & $6.0 \pm 2.7$ & $7.2 \pm 4.0$ & 1.0 & 0.6 & 0.5 & 2.6 & 1.9 & 1.5 \\
\hline $\begin{array}{l}\text { Lenses stained } \\
\text { with anti- } \\
\text { laminin-1 }\end{array}$ & $5.0 \pm 2.4$ & $3.3 \pm 1.3$ & $4.1 \pm 2.3$ & 0.7 & 0.5 & 1.1 & 2.3 & $2.0^{(*)}$ & $2.4^{(*)}$ \\
\hline
\end{tabular}


Table 2: Medians of statistical estimators (bootstrap) of grain size probability distribution (mean, standard deviation, skewness and kurtosis) of the retinas marked with anti-collagen IV or anti-laminin-1 from control and FA deficient groups D2 and D8. (*): not significant difference in estimator probability distribution.

\begin{tabular}{|l|c|c|c|c|c|c|c|c|c|}
\cline { 2 - 9 } \multicolumn{1}{c|}{} & \multicolumn{3}{c|}{ Mean \pm std } & \multicolumn{3}{c|}{ Mean Skewness } & \multicolumn{3}{c|}{ Mean Kurtosis } \\
\cline { 2 - 9 } \multicolumn{1}{c|}{} & Control & D2 & D8 & Control & D2 & D8 & Control & D2 & D8 \\
\hline $\begin{array}{l}\text { Retinas } \\
\text { stained with } \\
\text { anti-Collagen } \\
\text { IV }\end{array}$ & $3.3 \pm 1.1$ & $4.0 \pm 1.7$ & $4.7 \pm 2.6$ & 0.5 & 0.6 & 0.8 & 2.5 & $2.6^{(*)}$ & $2.4^{(*)}$ \\
\hline $\begin{array}{l}\text { Retinas } \\
\text { stained with } \\
\text { anti-laminin- } \\
1\end{array}$ & $2.5 \pm 1.3$ & $3.5 \pm 1.4$ & $3.0 \pm 1.1$ & 1.4 & 0.8 & 0.8 & 3.4 & 2.5 & 2.6 \\
\hline
\end{tabular}

